\title{
Comparative Study of Si-Ge-Sn Resonant Cavity Enhanced Heterojunction Bipolar Phototransistor (RCE-HPT) under Quantum Confined Stark Effect (QCSE) and Franz Keldysh Effect (FKE) at $1.55 \mu \mathrm{m}$
}

Soumava Ghosh ( $\sim$ ghoshsoumava2@gmail.com )

Institute of Radiophysics and Electronics

Research Article

Keywords: Franz Keldysh Effect, Quantum Confined Stark Effect, RCE HPT

Posted Date: July 16th, 2021

DOl: https://doi.org/10.21203/rs.3.rs-600692/v1

License: (9) This work is licensed under a Creative Commons Attribution 4.0 International License.

Read Full License 


\title{
Comparative Study of Si-Ge-Sn Resonant Cavity Enhanced Heterojunction Bipolar Phototransistor (RCE-HPT) under Quantum Confined Stark Effect (QCSE) and Franz Keldysh Effect (FKE) at $1.55 \mu \mathrm{m}$
}

\author{
Soumava Ghosh \\ Institute of Radio Physics and Electronics, 92 A. P. C Road, Kolkata-700009 \\ ghoshsoumava2@gmail.com
}

\begin{abstract}
Group-IV and their alloy based Heterojunction Bipolar Phototransistors (HPTs) are of immense interest in recent day optical communication. In this paper first resonant cavity enhanced heterojunction bipolar phototransistor (RCE-HPT) with $\mathrm{Ge}_{0.992} \mathrm{Sn}_{0.008} / \mathrm{Si}_{0.30} \mathrm{Ge}_{0.61} \mathrm{Sn}_{0.09}$ Quantum Well/barrier structure under Quantum Confined Stark Effect (QCSE) has been evaluated. Further the bulk GeSn absorption region has been considered instead of QW/barrier structure and estimated the Franz Keldysh Effect (FKE). Finally different RCE-HPT related parameters such as quantum efficiency-bandwidth product, responsivity, collector current and optical gain have been studied and compared under QCSE and FKE.
\end{abstract}

Key Words: Franz Keldysh Effect, Quantum Confined Stark Effect, RCE HPT

\section{Introduction}

Over last few decades, optical communication has attracted new data hungry generation due to its low loss, low attenuation and high speed than conventional electronic communication. Among different active optical devices, phtotodetector (PD) is an important building block. In last few years a large number of researches have been done on PDs with III-V materials and their alloy. Although their performance is excellent in optical communication [1], but high fabrication cost has restricted their applications. Therefore, researchers have shifted their focus to alternative group-IV semiconductors. Si is an elementary group-IV semiconductor, but it is not compatible with modern optical communication window $(1.3 \mu \mathrm{m}$ and $1.55 \mu \mathrm{m})$, due to its bandgap energy i.e, $1.12 \mathrm{eV}$ [2]. This problem can be partially eradicated by using Ge, as its direct bandgap 
supports $1.55 \mu \mathrm{m}$ at room temperature. But beyond $1.5 \mu \mathrm{m}$ the optical response of Ge based devices falls drastically [3]. Therefore, entire optical telecommunication bands cannot be covered by them.

This scenario has been changed dramatically in last few decades after successful growth of high quality $\mathrm{Ge}_{1-\mathrm{y}} \mathrm{Sn}_{\mathrm{y}}$ films and $\mathrm{SiGeSn}$ alloys on $\mathrm{Si}$ substrate by practical chemical vapor deposition (CVD) [4,5] and molecular beam epitaxy (MBE) [6]. Beyond 8\% Sn concentration the $\mathrm{Ge}_{1-\mathrm{y}} \mathrm{Sn}_{\mathrm{y}}$ alloy becomes a direct bandgap material [7] and further enhancement of Sn concentration reduces the bandgap and not only increases the absorption coefficient but also shows a red shift [8]. Based on those notable features researchers have developed different types of optical devices such as, light emitting diode [9], LASER [10,11], p-i-n PD [12-15], metal-semiconductor-metal PD (MSM PD) [16], avalanche photodiode (APD) [17], waveguide PD [18], quantum well infrared photodetector (QWIP) [19-22] and heterojunction bipolar phototransistor (HPT) [8, 2327].

Among all those aforesaid PDs, HPT is a potential device. It is a strong competitor of p-i-n and APD due to its inherent gain and absence of excess noise. A number of researchers have reported the characteristics of HPT based on III-V compounds and their alloys [28-32] and group-IV compounds [23-27]. Basu et al. [23] have reported their study on HPT based on GeSn, which shows that GeSn based HPT can compete with InGaAs based devices.

Fidaner et al. [33] and Basu and Das [34] have reported the characteristics of photoconductors exploiting Quantum Confined Stark Effect (QCSE) and Franz-Keldysh Effect (FKE) respectively. Chang and Chang [35] have reported a strain free, type-I structure of $\mathrm{Ge}_{0.992} \mathrm{Sn}_{0.008}$ quantum well $(\mathrm{QW})$ with $\mathrm{Si}_{0.30} \mathrm{Ge}_{0.61} \mathrm{Sn}_{0.09}$ barrier. The lowest bandgap of $\mathrm{QW}$ is direct in nature reduces to $773.41 \mathrm{meV}$, which is lower than the bandgap of pure Ge. Chakraborty et al. [36] have shown resonant cavity enhanced (RCE) p-i-n PD, exploiting both FKE and QCSE. In our previous work [25] we have reported a comparison of HPT with and without resonant cavity. The RCE-HPT shows approximately eight times better response than conventional HPT with equal thickness of absorption layer. The multi pass of the light wave inside the device increases the absorption in the active layer and thereby the optical response. These findings encourage the author for extending the study on RCE based HPT under QCSE and FKE and also comparing their performance at the recent telecommunication window i.e, $1.55 \mu \mathrm{m}$. 
In this paper different RCE-HPT parameters exploiting FKE and QCSE such as Quantum Efficiency-Bandwidth Product (QEBWP), responsivity, terminal current, optical gain etc have been studied. The proposed structure exhibits in sec. 2, theoretical analysis part presents in sec. 3, followed by analytical results in sec. 4 . Finally, the conclusion of this study presents in sec. 5 .

\section{Structure}

In this study a $N-p-n$ HPT with $100 \mu \mathrm{m}^{2}$ cross-sectional area has been considered with normal biasing technique. The transverse electric (TE or $\mathrm{x}-\mathrm{y}$ ) polarized light incident on the emitter-base junction, i.e., front side illumination has been considered. Fig. 1 shows the proposed schematic HPT structure, which is grown on Si substrate via a fully strain-relaxed and lattice matched $\mathrm{Si}_{0.30} \mathrm{Ge}_{0.61} \mathrm{Sn}_{0.09}$ buffer layer. The material compositions, doping concentrations, bandgap and dimensions of different regions are given in Table $1.11 \mathrm{~nm}$ thick low bandgap $\mathrm{Ge}_{0.992} \mathrm{Sn}_{0.008} \mathrm{QW}$ with $10 \mathrm{~nm}$ thick higher bandgap $\mathrm{Si}_{0.30} \mathrm{Ge}_{0.61} \mathrm{Sn}_{0.09}$ barriers are used for absorption region, which is sandwiched between base and collector region. Higher bandgap $n$-type $\mathrm{Si}_{0.34} \mathrm{Ge}_{0.56} \mathrm{Sn}_{0.10}$ has been considered as the emitter. The top and bottom mirror with reflectivity $R_{1}$ and $R_{2}$ have been used to create a vertical cavity effect. Under strong external electric field this structure shows QCSE phenomenon. On the other hand, if the absorption region is replaced by equal thickness i.e, $31 \mathrm{~nm}$ of bulk $\mathrm{Ge}_{0.992} \mathrm{Sn}_{0.008}$, in the presence of electric field it shows a strong FKE.

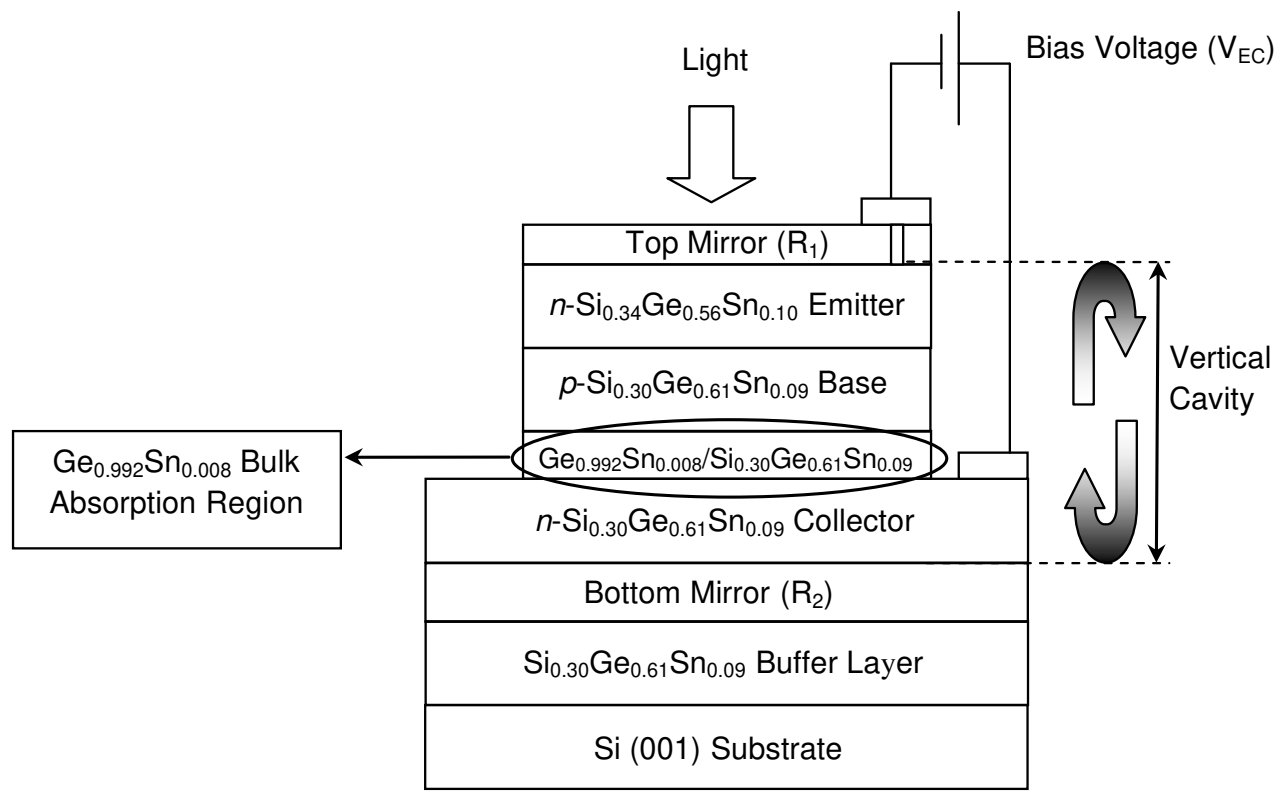

Fig. 1 Schematic diagram of front-side-illuminated SiGeSn HPT. 
Table 1: Different parameters of SiGeSn HPT

\begin{tabular}{|c|c|c|c|c|c|}
\hline Layer & Material & Band gap (eV) & Doping $\left(/ \mathbf{m}^{3}\right)$ & Type & $\begin{array}{l}\text { Width } \\
\text { (nm) }\end{array}$ \\
\hline Emitter & $\mathrm{Si}_{0.34} \mathrm{Ge}_{0.56} \mathrm{Sn}_{0.10}$ & 0.9483 & $1 \times 10^{24}$ & $N$ & 200 \\
\hline Base & $\mathrm{Si}_{0.30} \mathrm{Ge}_{0.61} \mathrm{Sn}_{0.09}$ & 0.9144 & $2 \times 10^{24}$ & $p$ & 200 \\
\hline \multirow{2}{*}{$\begin{array}{l}\text { Absorption } \\
\text { Region }\end{array}$} & $\mathrm{Ge}_{0.992} \mathrm{Sn}_{0.008}(\mathrm{FKE})$ & 0.6495 & \multirow[t]{2}{*}{ Undoped } & \multirow[t]{2}{*}{-} & 31 \\
\hline & $\begin{array}{l}\mathrm{Ge}_{0.992} \mathrm{Sn}_{0.008} / \\
\mathrm{Si}_{0.30} \mathrm{Ge}_{0.61} \mathrm{Sn}_{0.09}(\mathrm{QCSE})\end{array}$ & $\begin{array}{l}0.6495-\text { QW } \\
0.9144-\text { Barrier }\end{array}$ & & & $\begin{array}{l}11-\mathrm{QW} \\
10-\text { Barrier }\end{array}$ \\
\hline Collector & $\mathrm{Si}_{0.30} \mathrm{Ge}_{0.61} \mathrm{Sn}_{0.09}$ & 0.9144 & $5 \times 10^{22}$ & $n$ & 500 \\
\hline
\end{tabular}

\section{Theoretical Modeling}

In this section, the theoretical modeling of the proposed RCE-HPT, including the parameters related to absorption coefficient, quantum efficiency $(\mathrm{QE})$, responsivity, bandwidth (BW), terminal current and optical gain are given. The parameters related to GeSn and SiGeSn alloys are obtained by linear interpolation of these of Si, Ge and $\mathrm{Sn}$ which can be found in Ref. [10].

\subsection{Absorption Coefficient Calculation}

Due to the physical phenomenon QCSE, the absorption coefficient may be expressed as [36],

$$
\begin{aligned}
& \alpha(\hbar \omega)=\alpha_{\mathrm{h}} \exp \left[-\frac{\left(\hbar \omega-\hbar \Omega_{\mathrm{h}}\right)^{2}}{2\left(\hbar \Gamma_{\mathrm{h}}\right)^{2}}\right]+\alpha_{\mathrm{l}} \exp \left[-\frac{\left(\hbar \omega-\hbar \Omega_{\mathrm{l}}\right)^{2}}{2\left(\hbar \Gamma_{\mathrm{l}}\right)^{2}}\right] \\
&+\frac{\alpha_{\mathrm{c}}}{1+\exp \left(\frac{\hbar \Omega_{\mathrm{c}}-\hbar \omega}{\hbar \Gamma_{\mathrm{c}}}\right)} \times \frac{2}{1+\exp \left\{2 \pi\left[\left|\hbar \Omega_{\mathrm{c}}-\hbar \omega\right| / \mathrm{R}_{\mathrm{y}}\right]^{-1 / 2}\right\}}
\end{aligned}
$$

where, the optical energy is $\hbar \omega$, the excitonic peak energy is $\hbar \Omega$, $\Gamma$ 's are the line width (HWHM), $\alpha$ 's represent the fitting parameters, $\mathrm{R}_{\mathrm{y}}$ is the Rydberg's constant for exciton. The subscripts h, 1 and c denote heavy hole (HH), light hole (LH) and continuum states respectively.

For the bulk absorption region, the absorption coefficient due to FKE is given below [36],

$$
\alpha(\hbar \omega)=\frac{\alpha_{\mathrm{b}}}{(2 \pi)^{2}}\left[\frac{2 \mu_{\mathrm{r}}}{\hbar^{2}}\right]^{3 / 2}\left(\hbar \omega-\mathrm{E}_{\mathrm{g}}\right)^{1 / 2} \times\left[1-\frac{\mathrm{E}_{0}^{3 / 2}}{\left(\hbar \omega-\mathrm{E}_{\mathrm{g}}\right)^{3 / 2}} \cos \left\{\frac{4}{3}\left[\frac{\left(\hbar \omega-\mathrm{E}_{\mathrm{g}}\right)}{\mathrm{E}_{0}}\right]^{3 / 2}\right\}\right]
$$


where, $E_{g}, \mu_{r}$ and $E_{0}$ stand for direct band gap energy, reduced mass and subband energy respectively.

By using the approach of model solid theory [37], the band gap energy $E_{\mathrm{g}}$ is obtained by [36],

$\mathrm{E}_{\mathrm{g}}=\mathrm{E}_{\mathrm{dir}}+\delta \mathrm{E}_{\mathrm{h}}^{\mathrm{c} \Gamma}$ and $\delta \mathrm{E}_{\mathrm{h}}^{\mathrm{c} \Gamma}=\mathrm{a}_{\mathrm{c}} \frac{\Delta \Omega}{\Omega}$ where $\mathrm{a}_{\mathrm{c}}$ is the conduction band hydrostatic deformation potential and $\mathrm{E}_{\mathrm{dir}}$ denote the direct band gap of the absorption region.

The sub band energy $\left(E_{0}\right)$ and the absorption coefficient $\left(\alpha_{b}\right)$ depend on the external electric field F, are given below [36],

$$
\alpha_{\mathrm{b}}=\frac{2 \pi\left\langle\left|\mathrm{p}_{\mathrm{cv}}\right|^{2}\right\rangle}{\mathrm{n} \varepsilon_{0} \omega \mathrm{c}}\left(\frac{\mathrm{e}}{\mathrm{m}_{0}}\right)^{2} \text { and } \mathrm{E}_{0}=\left(\mathrm{e}^{2} \mathrm{~F}^{2} \hbar^{2} / 2 \mu_{\mathrm{r}}\right)^{1 / 3}
$$

where, the momentum matrix is,

$$
\left\langle\left|p_{c v}\right|^{2}\right\rangle=\frac{m_{0}^{2} E_{g}\left(E_{g}+\Delta\right)}{3 m_{e}\left(E_{g}+2 \Delta / 3\right)}
$$

$\Delta$ is the spin-orbit splitting and $\mathrm{n}$ is the refractive index (R.I.).

\subsection{Photodetector parameters}

For a conventional structure without any resonating cavity, the QE can be expressed as [25],

$\eta_{\text {Conventional-HPT }}=\left(1-\mathrm{R}_{1}\right)\left(1-\mathrm{e}^{-\alpha \mathrm{d}}\right)$

where, $\alpha$ is the absorption coefficient, $d$ is the active region thickness and $R_{1}$ is the reflectivity of the anti-reflection (AR) coating for reducing light reflection from the top surface of the HPT.

The QE of the resonant cavity structure is given as [25],

$\eta_{\text {RCE-HPT }}=\frac{\left(1+\mathrm{R}_{2} \mathrm{e}^{-\alpha \mathrm{d}}\right)\left(1-\mathrm{R}_{1}\right)\left(1-\mathrm{e}^{-\alpha \mathrm{d}}\right)}{1-2 \sqrt{\mathrm{R}_{1} \mathrm{R}_{2}} \mathrm{e}^{-\alpha \mathrm{d}} \cos \left(2 \beta \mathrm{L}+\Psi_{1}+\Psi_{2}\right)+\mathrm{R}_{1} \mathrm{R}_{2} \mathrm{e}^{-2 \alpha \mathrm{d}}}$

where, $\alpha(\mathrm{E}, \mathrm{F})$ is the absorption coefficient at photon energy $\mathrm{E}$ and external electric field $\mathrm{F}, \mathrm{L}$ is the cavity length, $\Psi_{1}$ and $\Psi_{2}$ are the phase shifts introduced by top and bottom mirror, and $\mathrm{R}_{1}$ and $\mathrm{R}_{2}$ are the front and back mirror reflectivity respectively. The propagation constant $(\beta)$ is 
calculated by, $\beta=\frac{2 \pi n}{\lambda}$, where $\lambda$ is the wavelength and $n$ is the R.I. As, the R.I. (n) changes to (n $+\Delta \mathrm{n})$, due to the linear electro optic effect, so the propagation constant $(\beta)$ and QE $(\eta)$ change according to the external electric field.

The BW of the PD depends on both RC and transit time delay components. As the active region is very thin, in this analysis the RC time delay can be neglected. Therefore, the BW can be calculated by using the relation [25],

$\mathrm{f}_{3-\mathrm{dB}}=0.45\left(\frac{\mathrm{v}}{\mathrm{d}}\right)$

where, $\mathrm{v}$ is the electron drift velocity [25],

$\mathrm{V}=\frac{\mu \mathrm{F}}{\sqrt{1+\left(\frac{\mu \mathrm{F}}{\mathrm{v}_{\mathrm{s}}}\right)^{2}}}$

where $\mu$ is the mobility and $v_{s}$ is the electron saturation velocity. Since, till now the saturation velocity of GeSn alloy is unknown; therefore, in this analysis the saturation velocity of pure Ge i.e, $6 \times 10^{4} \mathrm{~m} / \mathrm{s}$ has been considered [14]. As, GeSn alloy has higher carrier mobilities than pure Ge [38], the consideration of the value of saturation velocity of pure Ge may be true and reasonable.

The peak QE at resonant point, may be expressed as [39],

$\eta_{P}=\left\{\frac{\left(1+R_{2} e^{-\alpha d}\right)}{\left(1-\sqrt{R_{1} R_{2}} e^{-\alpha d}\right)^{2}}\right\} \times\left(1-R_{1}\right) \times\left(1-e^{-\alpha d}\right)$

The base-collector junction responsivity can be calculated by [14],

$\Re=\eta\left(\frac{\mathrm{e} \lambda}{\mathrm{hc}}\right)$

\subsection{HPT Modeling}

The common emitter current gain is obtained by [24], 
$\mathrm{h}_{\mathrm{FE}}=\frac{\alpha}{1-\alpha}=\frac{\gamma}{1+\gamma\left[\cosh \left(\frac{\mathrm{W}_{\mathrm{B}}}{\mathrm{L}_{\mathrm{nB}}}\right)-1\right]}$

where,

$\gamma=\frac{\gamma_{0}\left(\xi_{1} / \xi_{2}\right)}{\sinh \left(\mathrm{W}_{\mathrm{B}} / \mathrm{L}_{\mathrm{nB}}\right)}$

and,

$\gamma_{0}=\frac{\mathrm{D}_{\mathrm{nB}}}{\mathrm{D}_{\mathrm{pE}}} \frac{\mathrm{L}_{\mathrm{pE}}}{\mathrm{L}_{\mathrm{nB}}} \frac{\mathrm{N}_{\mathrm{E}}}{\mathrm{N}_{\mathrm{B}}}\left(\frac{\mathrm{m}_{\mathrm{nB}}^{*} \mathrm{~m}_{\mathrm{pB}}^{*}}{\mathrm{~m}_{\mathrm{nE}}^{*} \mathrm{~m}_{\mathrm{pE}}^{*}}\right) \exp \left(\frac{\Delta \mathrm{E}_{\mathrm{g}}}{\mathrm{k}_{\mathrm{B}} \mathrm{T}}\right)$

The responsivity of HPT can be calculated by [40],

$\mathcal{R}=\mathrm{h}_{\mathrm{FE}} \times \Re$

where $h_{F E}$ is the common emitter current gain and $\Re$ is the base-collector junction responsivity.

The enhancement factor (EF), which indicates the enhancement of the responsivity of the RCEHPT than conventional one can be calculated by [15],

$\mathrm{EF}=\mathcal{R}_{\mathrm{RCE}-\mathrm{HPT}} / \mathcal{R}_{\text {Conventional-HPT }}$

The collector current can be expressed as [25],

$\mathrm{I}_{\mathrm{C}}=\left(1+\mathrm{h}_{\mathrm{FE}}\right)\left(\mathrm{I}_{\mathrm{ph}}+\mathrm{I}_{\mathrm{CO}}\right)$

where IC0 stands for the reverse saturation current, can be written as [25],

$\mathrm{I}_{\mathrm{C} 0}=\mathrm{eA}\left[\frac{\mathrm{D}_{\mathrm{pE}}}{\mathrm{L}_{\mathrm{pE}}} \mathrm{p}_{\mathrm{n} 0}+\frac{\mathrm{D}_{\mathrm{nB}}}{\mathrm{L}_{\mathrm{nB}}} \mathrm{n}_{\mathrm{p} 0} \tanh \left(\frac{\mathrm{W}_{\mathrm{B}}}{\mathrm{L}_{\mathrm{nB}}}\right)\right]$

where, $\mathrm{D}$ and $\mathrm{L}$ denote the diffusivity constant and diffusion length of the carrier, $\mathrm{W}$ is the thickness, $\mathrm{A}$ is the cross-sectional area, $\mathrm{n}_{\mathrm{p} 0}$ and $\mathrm{p}_{\mathrm{n} 0}$ are the minority carrier concentrations in the emitter and base under thermal equilibrium condition. The subscripts $\mathrm{p}, \mathrm{n}, \mathrm{E}$ and $\mathrm{B}$ stand for hole, electron, emitter and base respectively.

$\mathrm{I}_{\mathrm{ph}}$ is the photocurrent, is obtained by [25],

$\mathrm{I}_{\mathrm{ph}}=\Re \times \mathrm{P}_{\mathrm{inc}}$

where $\mathrm{P}_{\text {inc }}$ is the incident photon power. 
The optical gain (G) can be defined as [25],

$$
\mathrm{G}=\left(1+\mathrm{h}_{\mathrm{FE}}\right) \times \eta
$$

\section{Results and Discussions}

With the help of eqn. (1) the absorption spectra in the presence of QCSE phenomenon has been evaluated. The different parameters regarding this calculation are taken from the Ref. [41]. When a bulk $\mathrm{Ge}_{0.992} \mathrm{Sn}_{0.008}$ absorption layer has been considered instead of $\mathrm{GeSn} / \mathrm{SiGeSn}$ well-barrier structure, then a strong FKE has been noticed in the presence of external electric field. The absorption coefficient under FKE has been calculated by eqn. (2). Those values of absorption coefficients are used for $\mathrm{QE}$ and responsivity calculation.

The QEBWP has been calculated by using eqns. (6) and (7). The velocity of the electron in eqn. (7) is the drift velocity, which is a simplified assumption at high speed [25]. The responsivity of HPT has been estimated by eqn. (14). In fig. 2 and 3 we have plotted the QEBWP and HPT responsivity against optical frequency (wavelength). The variation of relative $\mathrm{QE}(\Delta \eta / \eta)$ as a function of optical frequency (wavelength), which has been evaluated from eqns. (6) and (9) is shown in fig. 4. By using eqn. (15) the EF has been calculated and plotted against optical frequency (wavelength) in fig. 5. Fig. 6 depicts the variation of the calculated collector current (IC) measured by eqn. (16) with optical frequency (wavelength).

All the figures (fig. 2 to fig. 6) depict comparative studies of different parameters regarding HPT

exploiting QCSE and FKE where the operating voltage is fixed at $2 \mathrm{MV} / \mathrm{m}$. Those figures clearly exhibit that, the FKE based RCE-HPT show better response in lower optical frequency ranges of $178-192.8 \mathrm{THz}$ on the other hand QCSE becomes dominating in 192.8 to $203 \mathrm{THz}$ range.

The band gap of the bulk $\mathrm{Ge}_{0.992} \mathrm{Sn}_{0.008}$ is $0.74 \mathrm{eV}$ which supports $1.68 \mu \mathrm{m}(\sim 178 \mathrm{THz})$ wavelength and therefore does not match with the recent optical communication window i.e, $1.55 \mu \mathrm{m}$. On the other hand, from those figures it has been noticed that at $\sim 192.8 \mathrm{THz}$ optical frequency which is equivalent to the $1.55 \mu \mathrm{m}$ wavelength QCSE shows approximately three times better response than FKE based RCE-HPT. So we can say that at $1.55 \mu \mathrm{m}$ QCSE based device is suitable than FKE. 
By using eqns. (11) and (19) the small signal current gain and optical gain have been calculated respectively. From the fig. 7 it has been concluded that the enhancement of emitter width can not affect the small signal current gain and optical gain of HPT at fixed carrier density in emitter, ie, $\mathrm{N}_{\mathrm{E}}=2 \times 10^{24} / \mathrm{m}^{3}$. The fig. 8 shows the variation of the small signal current gain and optical gain with base width. When the base width increases then more number of injected carriers from the emitter are recombined inside the base before reaching to the collector. So automatically the gains reduce for a constant base concentration $\mathrm{N}_{\mathrm{B}}=1 \times 10^{24} / \mathrm{m}^{3}$.

Fig. 9 depicts the effect of emitter carrier concentration of the gain of the RCE-HPT under FKE and QCSE. In both the cases it has been found that enhancement of the carrier concentration inside the emitter region increases the density of the injected carriers towards the collector end through base. Therefore, the optical and small signal current gains increase with enhancement of the emitter concentration. On the other hand, when the carrier concentration of the base increases, most of the injected carriers from the emitter are recombined before reaching to the collector end. Thus, the optical and electrical responses diminish with enhancement of the base carrier concentration. This phenomenon is depicted in fig. 10. In those plots of gain variation against width and concentration of emitter and base regions, the operating wavelength is fixed at $1.55 \mu \mathrm{m}$.

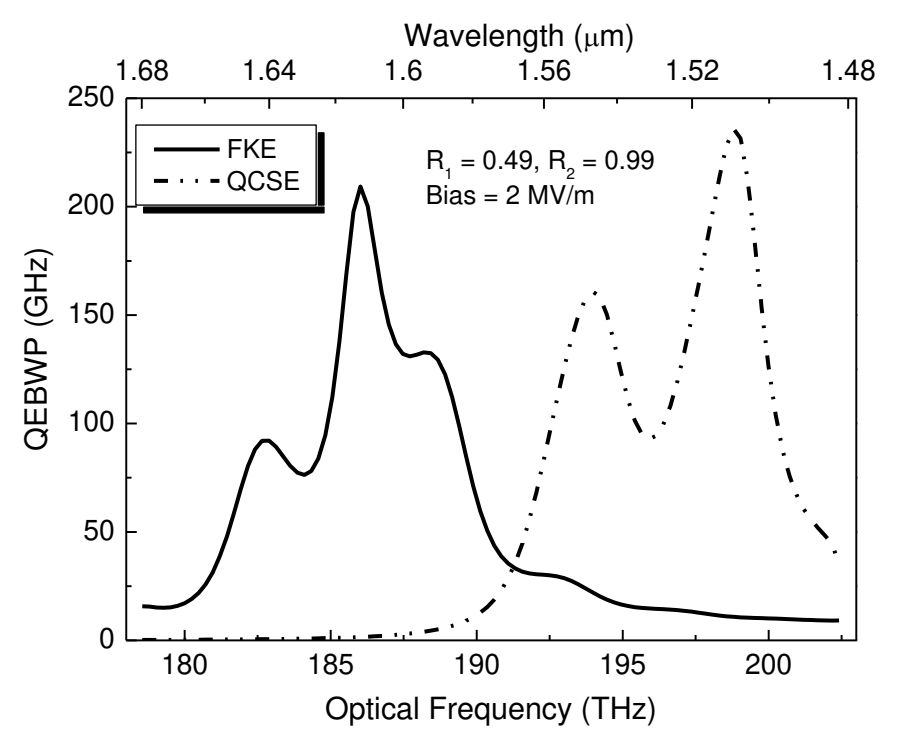

Fig. 2 QEBWP variation with Optical Frequency (Wavelength) for RCE-HPT. 


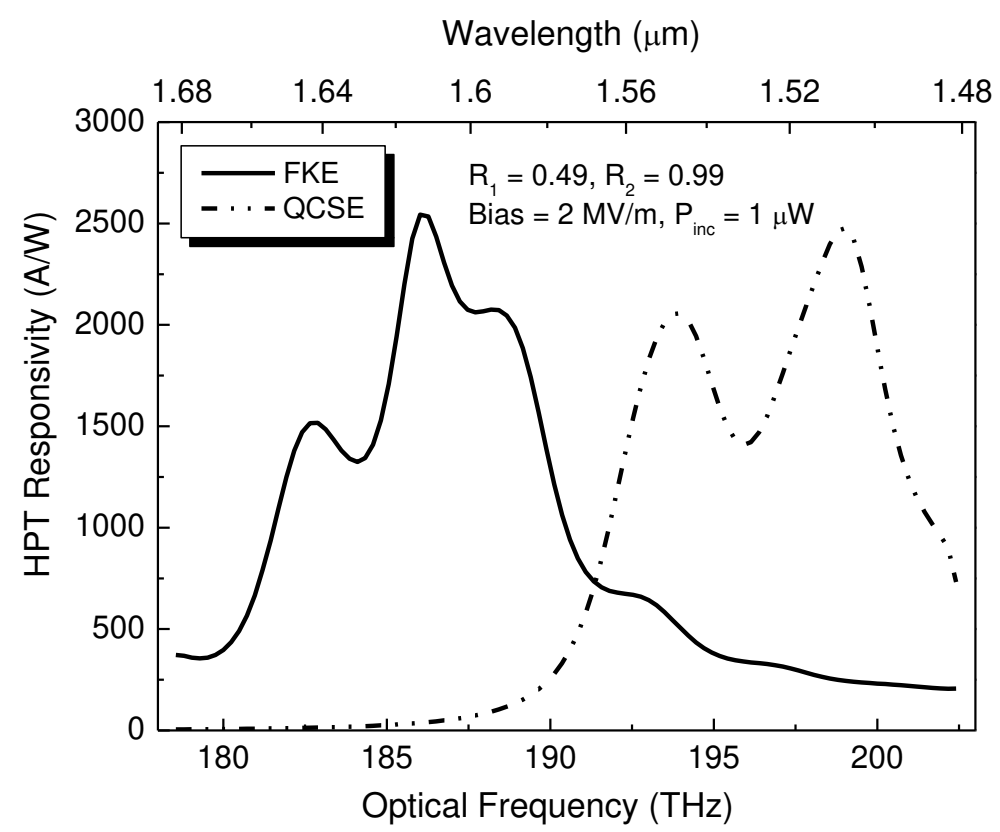

Fig. 3 Variation of HPT Responsivity with Optical Frequency (Wavelength) for RCE-HPT.

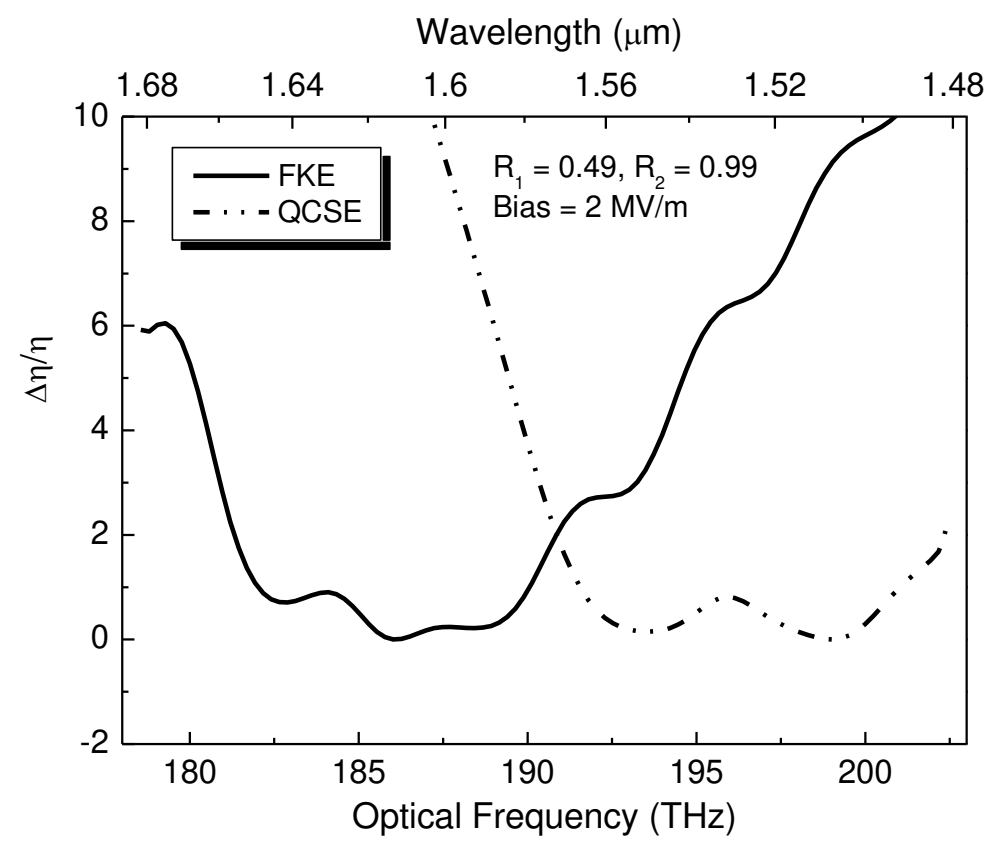

Fig. 4 Plot of $\Delta \eta / \eta$ with Optical Frequency (Wavelength) for RCE-HPT. 


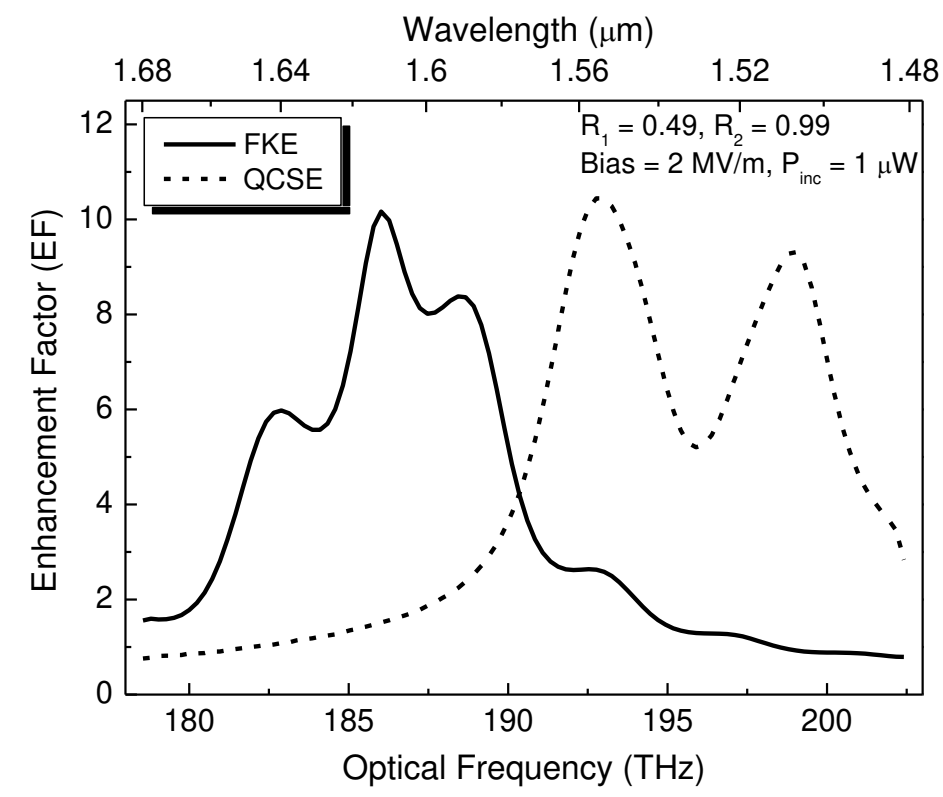

Fig. 5 Variation of Enhancement Factor against Optical Frequency (Wavelength) for RCE-HPT.

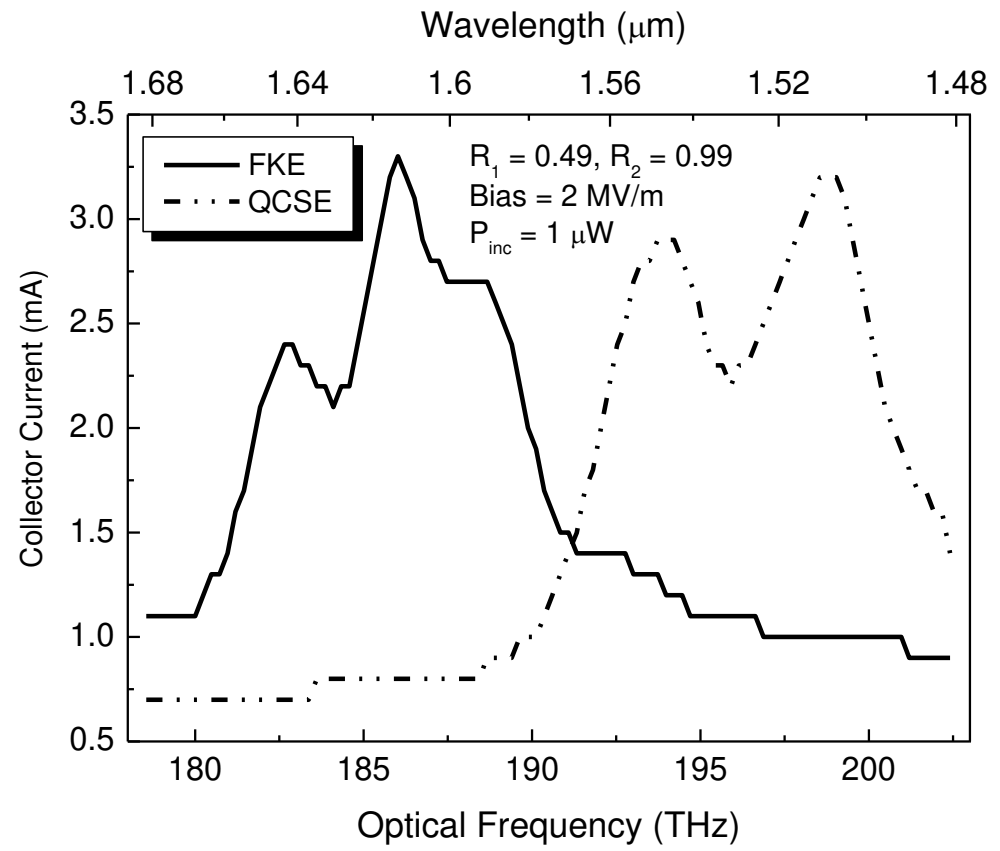

Fig. 6 Collector Current variation with Optical Frequency (Wavelength) for RCE-HPT. 


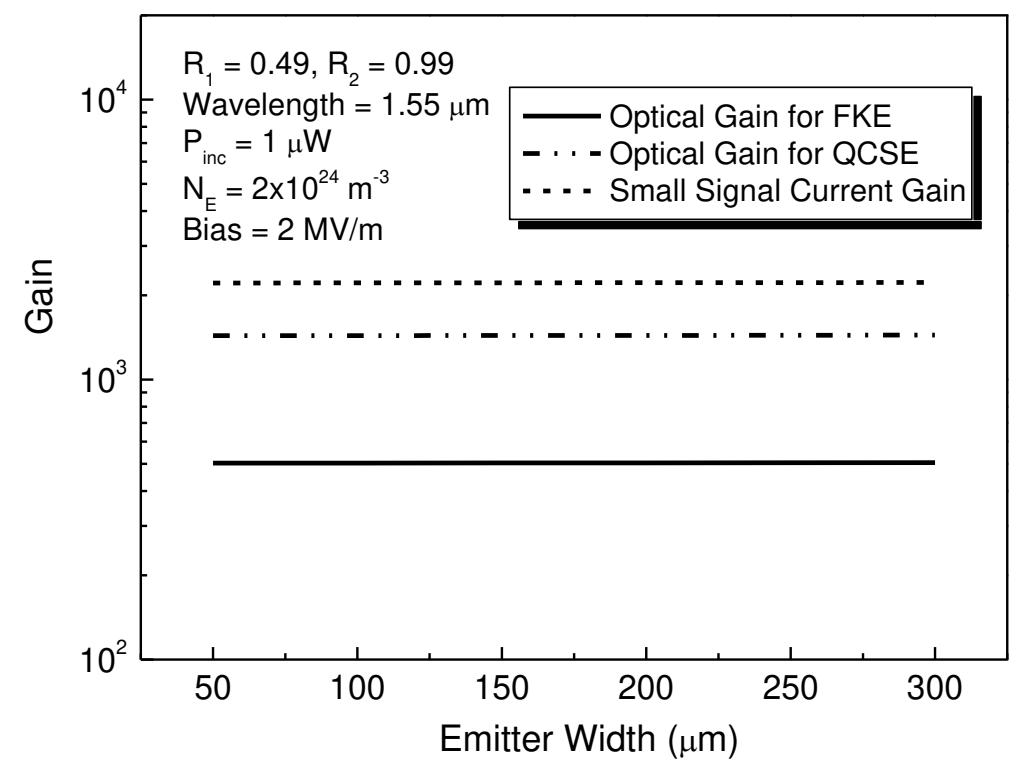

Fig. 7 Gain variation with Emitter Width for RCE-HPT.

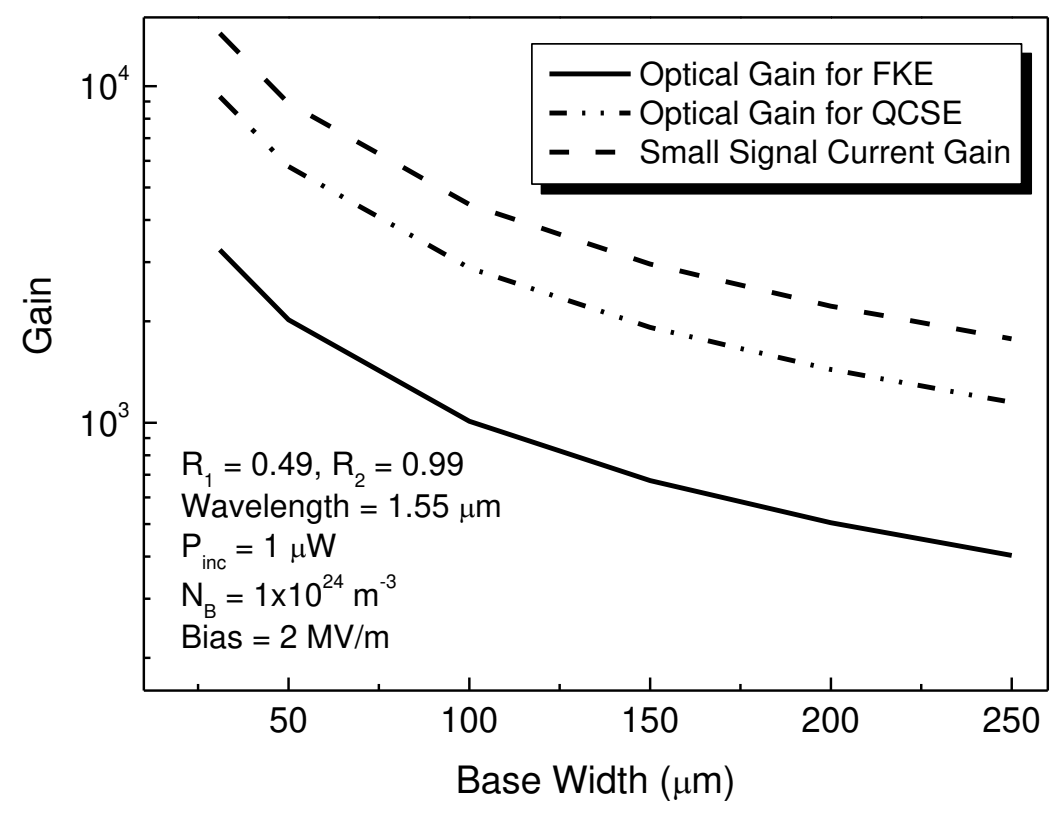

Fig. 8 Gain variation with Base Width for RCE-HPT. 


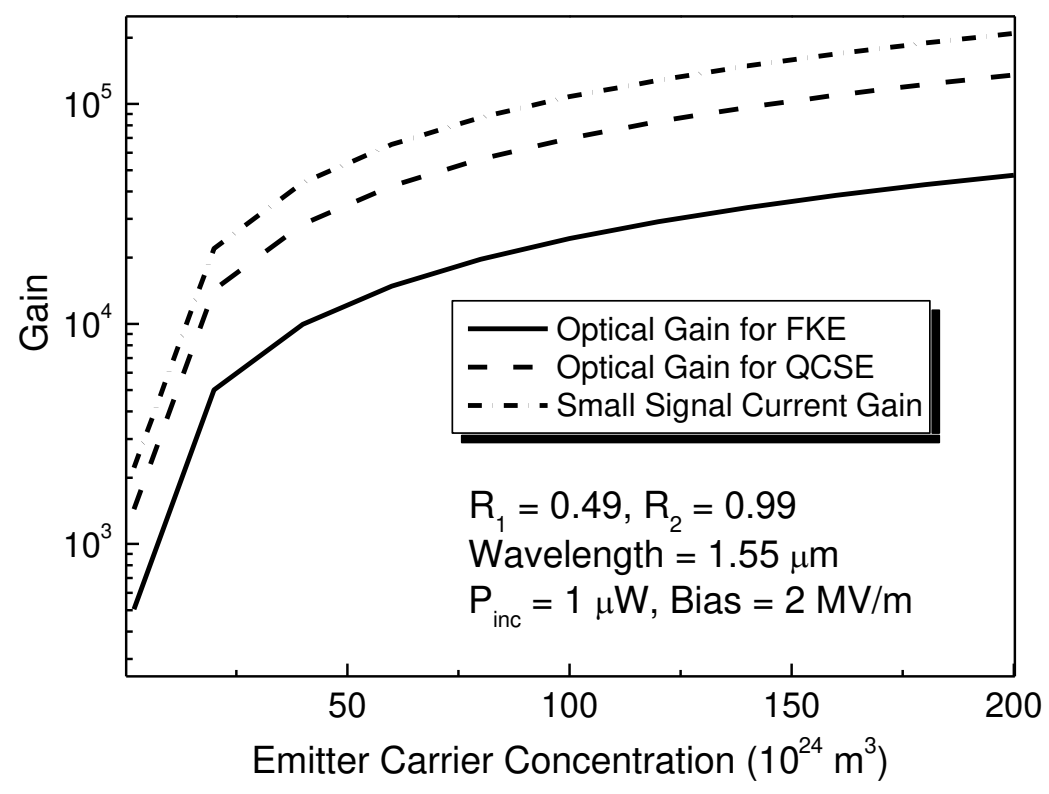

Fig. 9 Gain variation with Emitter Concentration for RCE-HPT.

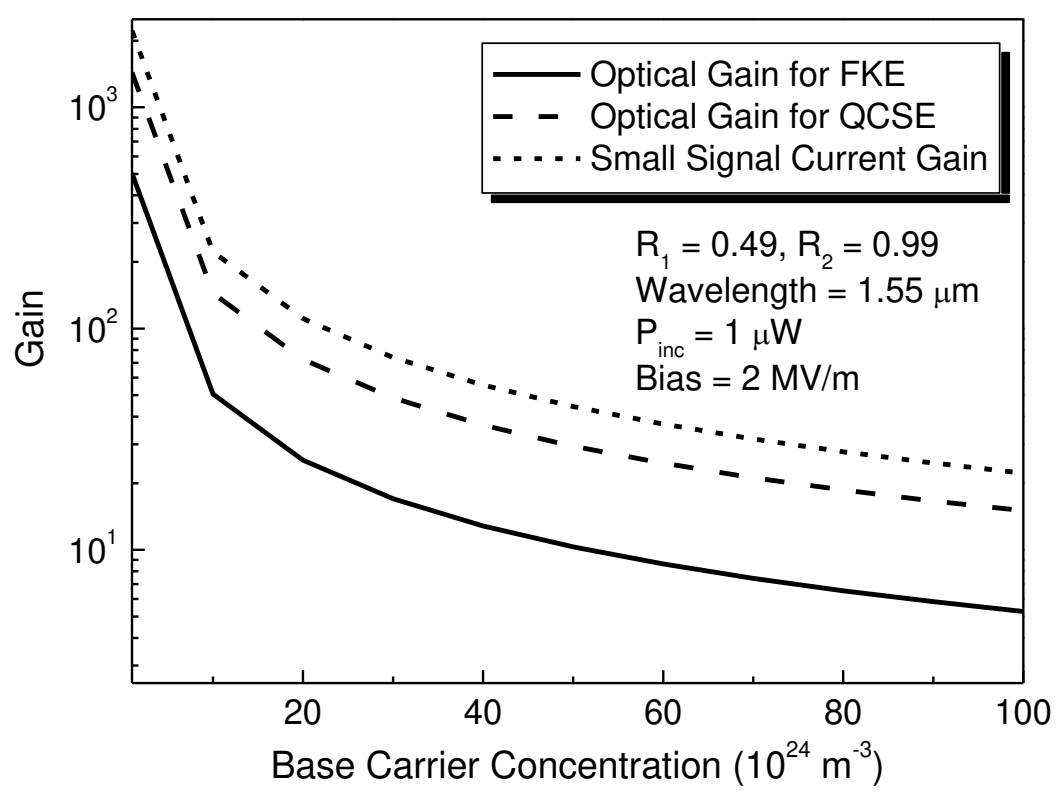

Fig. 10 Gain variation with Base Concentration for RCE-HPT. 


\section{Conclusion}

From this study it has been noticed that, when the absorption region is considered the $\mathrm{GeSn} / \mathrm{SiGeSn}$ well-barrier structure, the RCE-HPT shows a strong QCSE under external electric field. Again, when this structure has been replaced by equivalent thickness of bulk GeSn, due to the direct band gap nature and high absorption it shows FKE in the presence of electric field. In this study first different characteristics of RCE-HPT exploiting strong QCSE and FKE have been compared. After that at $1.55 \mu \mathrm{m}$ the optical and small signal current gains have been evaluated as a function of width and carrier concentration of the base and emitter region. Though the band gap of the GeSn does not match with $1.55 \mu \mathrm{m}$ but nevertheless at that wavelength QCSE based device gives approximately three times better response than FKE based RCE-HPT. It requires further experimental and analytical study to get proper $\mathrm{Sn}$ composition in Ge for exploiting FKE and QCSE correctly at $1.55 \mu \mathrm{m}$.

\section{References}

1. R. W. M. Hoogeveen, R. J. van der A, and A. P. H. Goede: Extended wavelength InGaAs infrared $(1.0-2.4 \mu \mathrm{m})$ detector arrays on SCIAMACHY for space-based spectrometry of the Earth atmosphere. Infr. Phys. Technol. 42, 1-16 (2001)

2. M. J. Deen, P. K. Basu: Silicon Photonics: Fundamentals and Devices. Wiley, Chichester (2012)

3. J. Michel, J. Liu, L. C. Kimerlin: Ge-on-Si Photodeectors. Nat. Photonics 4, 527-534 (2010)

4. M. Bauer, J. Taraci, J. Tolle, A.V.G. Chizmeshya, S. Zollner, D.J. Smith, J. Menendez, C. Hu, J. Kouvetakis: Ge-Sn semiconductors for band-gap and lattice engineering. Appl. Phys. Lett. 81(1-3), 2992 (2002)

5. A. V. G. Chizmeshy, C. Ritter, J. Tolle, C. Cook, J. Menendez, J. Kouvetakis: Fundamental studies of $\mathrm{P}\left(\mathrm{GeH}_{3}\right)_{3}, \mathrm{As}\left(\mathrm{GeH}_{3}\right)_{3}$, and $\mathrm{Sb}\left(\mathrm{GeH}_{3}\right)_{3}$ : practical n-dopants for new group IV semiconductors. Chem. Mater. 18, 6266-6277 (2006)

6. J. P. Gupta, N. Bhargava, S. Kim, T. Adam, and J. Kolodzey: Infrared electroluminescence from GeSn heterojunction diodes grown by molecular beam epitaxy. Appl. Phys. Lett. 102, 251117 (2013) 
7. B. Dutt, H. Lin, D. S. Sukhdeo, B. M. Vulovic, S. Gupta, D. Nam, K. C. Saraswat, J. S. Harris Jr.: Theoretical Analysis of GeSn Alloys as a Gain Medium for a Si-Compatible Laser. IEEE J. Sel. Top. Quant. Electron. 19, 1502706 (2013)

8. G. -E. Chang, R. Basu, B. Mukhopadhyay, and P. K. Basu: Design and modeling of GeSn-based heterojunction phototransistors for communication applications. IEEE J. Sel. Topics Quantum Electron. 22, 425-433 (2016)

9. M. Oehme, K. Kostecki, T. Arguirov, G. Mussler, K. Ye, M. Golhofer, M. Schmid, M. Kaschel, R. A. Körner, M. Kittler, et al.: GeSn Heterojunction LEDs on Si Substrates. IEEE Photon. Technol. Lett. 26, 187-189 (2014)

10. G. -E. Chang, S. -W. Chang, S. L. Chuang: Strain-Balanced $\mathrm{Ge}_{\mathrm{z}} \mathrm{Sn}_{1-\mathrm{z}}-\mathrm{Si}_{\mathrm{x}} \mathrm{Ge}_{\mathrm{y}} \mathrm{Sn}_{1-\mathrm{x}-\mathrm{y}}$ Multiple-Quantum-Well Lasers. IEEE J. Quantum Electron. 46, 1813-1820 (2010)

11. S. Ghosh, B. Mukhopadhyay, G. Sen: Performance Enhancement of GeSn Transistor Laser with Symmetric and Asymmetric Multiple Quantum Well in the Base. Semiconductors 54, 77-84 (2020)

12. S. Su, B. Cheng, C. Xue, W. Wang, et al.: GeSn p-i-n photodetector for all telecommunication band detection. Opt. Express 19, 6400-6405 (2011)

13. M. Oeheme, M. Schmid, M. Kaschel, M. Gollhofer, D. Widmann, E. Kasper, J. Schulze: GeSn p-i-n detectors integrated on Si with up to 4\% Sn. Appl. Phys. Lett. 101, 141110 (2012)

14. S. Ghosh, B. Mukhopadhyay, G. -E. Chang: Design and Analysis of GeSn-Based Resonant-Cavity-Enhanced Photodetectors for Optical Communication Applications. IEEE Sens. J. 20, 7801-7809 (2020)

15. S. Ghosh, H. Kumar, B. Mukhopadhyay, G. -E. Chang: Design and Modeling of HighPerformance DBR-Based Resonant-Cavity-Enhanced GeSn Photodetector for FiberOptic Telecommunication Networks. IEEE Sens. J. 21, 9900-9908 (2021)

16. S. Ghosh, K. -C. Lin, C. -H. Tsai, H. Kumar, Q. Chen, L. Zhang, B. Son, C. S. Tan, M. Kim, B. Mukhopadhyay, and G. -E. Chang: Metal-Semiconductor-Metal GeSn Photodetectors on Silicon for Short-Wave Infrared Applications. Micromachines, 11, 795 (2020) 
17. Y. Dong, W. Wang, X. Xu, X. Gong, D. Lei, Q. Zhou, Z. Xu, W Khai Loke, S. -F. Yoon, G. Liang, Y. -C. Yeo: Germanium-Tin on Si Avalanche Photodiode: Device Design and Technology Demonstration. IEEE Trans. Electron. Dev. 62, 128-135 (2015)

18. Y. H. Peng, H. H. Cheng, V. I. Mashanov, G. -E. Chang: GeSn p-i-n waveguide photodetectors on silicon substrates. Appl. Phys. Lett. 105, 231109(1-4) (2014)

19. P. Pareek, M. K. Das, S. Kumar: Responsivity Calculation of Group IV-based Interband MQWIP. J. Comput. Electronics 17, 319-328 (2017)

20. S. Ghosh, S. Dey, B. Mukhopadhyay, G. Sen: Study of High-Frequency Performance in GeSn-Based QWIP. $7^{\text {th }}$ International Conf. Computers and Devices for Communication, 448-452 (2019)

21. S. Ghosh, B. Mukhopadhyay, G. Sen, P. K. Basu: Performance analysis of GeSn/SiGeSn Quantum Well Infrared Photodetector in Terahertz Wavelength Region. Phys. E 115, $113692(2020)$

22. S. Ghosh, A. Bhattacharyya, G. Sen, B. Mukhopadhyay: Optimization of different structural parameters of GeSn/SiGeSn Quantum Well Infrared Photodetector (QWIP) for low dark current and high responsivity. J. Comput. Electronics (in press). doi: 10.1007/s10825-021-01668-w

23. R. Basu, V. Chakraborty, B. Mukhopadhyay, P. K. Basu: Predicted performance of $\mathrm{Ge} / \mathrm{GeSn}$ hetero-phototransistors on Si substrate at $1.55 \mu \mathrm{m}$. Opt. Quant. Electron. 47, 387-399 (2015)

24. V. Chakraborty, S. Dey, R. Basu, B. Mukhopadhyay, P. K. Basu: Current gain and external quantum efficiency modeling of GeSn based direct bandgap multiple quantum well heterojunction phototransistor. Opt. Quant. Electron. 49(1-13), 125 (2017)

25. S. Ghosh, B. Mukhopadhyay, G. Sen, P. K. Basu: Study of Si-Ge-Sn based Heterobipolar Phototransistor (HPT) exploiting Quantum Confined Stark Effect and Franz Keldysh effect with and without resonant cavity. Phys. E 106, 62-67 (2019)

26. S. Ghosh, B. Mukhopadhyay, G. Sen, P. K. Basu: Analysis of Some Important Parameters of Si-Ge-Sn RCE-HPT Exploiting QCSE and FKE. In Proceedings of the URSI-RCRS, (2020) 
27. W. -T. Hung, D. Barshilia, R. Basu, H. H. Cheng, G. -E. Chang: Silicon-based Highresponsivity GeSn Short-wave Infrared Heterojunction Phototransistors with a Floating Base. Opt. Lett. 45, 1088-1091 (2020)

28. S. M. Frimel, K. P. Roenker: A thermionic-field-diffusion model for Npn bipolar heterojunction phototransistors. J. Appl. Phys. 82, 1427-1437 (1997)

29. S. M. Frimel, K. P. Roenker: Gummel-Poon model for Npn heterojunction bipolar phototransistor. J. Appl. Phys. 82, 3581-3592 (1997)

30. S. H. Tan, H. R. Clen, W. T. Chen, M. K. Hsu, A. H. Lin, W. S. Lour: Characterization and modeling of three-terminal heterojunction phototransistors using an InGaP layer for passivation. IEEE Trans. Electron. Dev. 52, 204-210 (2005)

31. H. A. Khan, A. A. Rezazadeh, S. Sohaib: Modeling and analysis of the spectral response for AlGaAs/GaAs HPTs for short wavelength optical communication: J. Appl. Phys. 109, 104507 (2011)

32. M. S. Park, J. H. Jang: Enhancement of optical gain in floating-base InGaP-GaAs heterojunction phototransistors. IEEE Photon. Technol. Lett. 22, 1202-1204 (2010)

33. O. Fidaner, A.K. Okyay, J.E. Roth, et al.: Ge-SiGe quantum well waveguide photodetectors on silicon for the near-infrared. IEEE Photon. Technol. Lett. 19(20), 16311633 (2007)

34. P. K. Basu, N. R. Das, et al.: Ge/Si photodetectors and group IV alloy based photodetector materials. Opt. Quant. Electron. 41, 567-581 (2009)

35. G. -E. Chang, C. O. Chang: Design of strain-free GeSn/SiGeSn quantum-well electroabsorption modulators at $1550 \mathrm{~nm}$ wavelength. IEEE $7^{\text {th }}$ International Conf. on Group IV Photonics, 87-89 (2010)

36. V. Chakraborty, B. Mukhopadhyay, P. K. Basu: Study of GeSn/SiGeSn RCE photodetectors based on Franz-Keldysh effect and quantum confined Stark effect. Opt. Quant. Electron. 47, 2381-2389 (2015)

37. C. G. Van de Walle, R. M. Martin: Theoretical calculations of heterojunction discontinuities in the Si/Ge system. Phys. Rev. B 34, 5621 (1986)

38. B. Mukhopadhyay, G. Sen, R. Basu, S. Mukhopadhyay, and P. K. Basu: Prediction of large enhancement of electron mobility in direct gap $\mathrm{Ge}_{1-\mathrm{x}} \mathrm{Sn}_{\mathrm{x}}$ alloy. Phys. Status Solidi $B, 254,1700244$ (2017) 
39. K. Kishino, M. S. Unlu, J. -I. Chyi, J. Reed, L. Arsenault, H. Morkoc: Resonant CavityEnhanced (RCE) photodetectors. IEEE J. Quant. Electron. 27(8), 2025-2034 (1991)

40. H. A. Khan: Spectral Response Modeling and Analysis of Heterojunction Bipolar Phototransistors. Ph. D Thesis The University of Manchester (2010)

41. V. Chakraborty, B. Mukhopadhyay, P. K. Basu: Performance prediction of an electro absorption modulator at $1550 \mathrm{~nm}$ using GeSn/SiGeSn Quantum Well structure. Phys. E 50, 67-72 (2013) 